\title{
RECENSIONI E SEGNALAZIONI
}

\author{
Tito Forcellese (a cura), 2 giugno. Nascita, storia e memorie della \\ Repubblica, 3: Geografie del voto e istituzioni, Roma, Viella, 2020, \\ pp. 331, euro 29,00 .
}

Nel 2020 è stata pubblicata, presso l'Editore Viella, una serie di volumi che costituiscono il frutto di un'ampia ed articolata ricerca collettiva promossa dalla SISSCO (Società italiana per lo studio della storia contemporanea), sotto la direzione di Maurizio Ridolfi, avente ad oggetto le vicende istituzionali, politiche, economiche, culturali e sociali riguardanti la nascita della Repubblica Italiana, sancita dal referendum del 2 giugno 1946. I singoli volumi sono dedicati, rispettivamente, a: 1. Il "momento repubblicano" nella costruzione della democrazia, a cura di M. Ridolfi; 2. Territorio, culture politiche e dinamiche sociali, a cura di S. Adorno; 4. L'Italia del 1946 vista dall'Europa, a cura di P. Dogliani e V. Galimi; 5. Immaginari, linguaggi e rituali, a cura di M. Ridolfi e M. Ravveduto; 6. I numeri del referendum istituzionale, a cura di M. Ridolfi e P. Totaro. In questa sede si recensirà solo il terzo, quello curato da T. Forcellese, dove compaiono, oltre alla introduzione di Forcellese stesso, i saggi di quest'ultimo sull'Abruzzo, di G. Schininà sulla Sicilia, di S. Mura sulla Sardegna, di G. Morese sulla Basilicata, di A. Bertolotti su Viterbo e la Tuscia, di E. Sellaroli e G. Zanibelli sulla Maremma, di M. Almagisti e M. Zanellato sul Veneto e la Toscana, di E. Colombo sulle province lombarde, di N. Tonietto sul Veneto, di A. Celi sulle Alpi sud-occidentali e di L. Tentoni sul voto nei capoluoghi di regione.

Come avviene sempre in rapporto ad opere di tal genere, e di natura pluridisciplinare, è difficile dar conto di tutti i risultati cui sono giunte le singole indagini, anche per la diversità dei metodi impiegati e delle fonti utilizzate dai diversi autori. Esito, questo, quasi scontato ove si tenga conto della diversa competenza scientifica degli studiosi coinvolti nell'ambizioso progetto di ricerca. Qui si confrontano storici contemporaneisti, storici delle istituzioni, storici economici, politologi, esperti di geografia politica ed elettorale, che seguono appunto, in modo più o meno coerente, i canoni e i paradigmi dei molteplici ambiti "accademici" di provenienza. In tale contesto spiccano, in particolare, i testi di SellaroliZanibelli e di Almagisti-Zanellato che tentano un approccio originale, di tipo statistico-economico e storico-politologico, nell'analisi del voto del 2 giugno 1946, e sui quali mi soffermerò più avanti proprio per la peculiarità dei loro contributi.

Quanto alle fonti, esse si dispiegano su un ventaglio ricco ed esaustivo di materiale documentario, composto - fra l'altro - da dati elettorali, ricavati da pubblicazioni ufficiali o da mirati scavi archivistici (centrali, locali e periferici) e poi aggregati (o disaggregati) ed elaborati in maniera originale; dalle risultanze della storiografia di riferimento e 
dall'utilizzo di cronache e memorie locali; dallo spoglio di riviste di settore e di periodici del tempo; dalla consultazione della bibliografia, recente o più risalente, in argomento.

I casi di studio presi in considerazione, e che vengono disposti e analizzati in ordine geografico ascendente per seguire le tappe cronologiche della "liberazione" dal fascismo avvenuta nelle varie zone, non coprono certo tutte le aree del Paese e quindi non troviamo, ad esempio, indagini specifiche su alcune regioni del Meridione (Calabria, Puglia) o del Centro-Italia (Emilia-Romagna, Umbria, Marche), ma questa carenza è ugualmente imputabile a molte delle ricerche, storiche e socio-economiche, effettuate "sul campo" ed a largo raggio, anche a causa della difficoltà di reperimento in loco di fonti esaurienti e facilmente accessibili o della reale disponibilità, sul posto, di studiosi competenti e adeguati a questo tipo di lavoro. In compenso, e a motivo di compiacimento, va segnalato che, a differenza di molta della letteratura precedente, ci si è qui soffermati e addentrati non solo sul piano nazionale, ma anche su quello periferico (comunale, provinciale), e che si è avviata una comparazione fra le tre diverse tornate elettorali che si susseguono nel corso del 1946: elezioni amministrative, per l'Assemblea Costituente, per il referendum istituzionale.

Non essendo possibile, in questa sede, pure per ragioni di competenza in materia, passare in rassegna, o anche solo riassumere, il contenuto dei singoli saggi, con il rischio di tornare quasi ogni volta sugli stessi argomenti, ritengo più utile incentrare l'attenzione su alcuni rilevanti snodi tematici, su talune questioni interpretative di particolare interesse che emergono dalla lettura dell'interessante volume qui esaminato.

Viene intanto confermata la "frattura" fra un Centro-Nord repubblicano ed un CentroSud monarchico, ma tale coincidenza e sovrapposizione non sempre si verifica, visto che anche al Sud si manifestano "macchie" a prevalenza repubblicana e al Nord non mancano "isole" monarchiche. Dunque emergono delle disomogeneità interne alle "macro-aree" sopra indicate, determinate, fra il resto, dal carattere policentrico della regione indagata (Abruzzo, Lombardia) o dalla appartenenza (politica, sociale, economica...) di taluni segmenti spaziali, compresi nei suoi confini, ad altre regioni limitrofe. Non meno rilevante è la dicotomia tra aree urbane e aree rurali, così come la distinzione fra grandi città e paesi più piccoli, ovvero la differente conformazione geofisica del territorio esaminato (zone pianeggianti, collinari, di montagna, valli insistenti su determinati bacini idrografici...) (Schininà, Forcellese, Colombo, Celi). Altra mancata coincidenza, ancorché limitata, riguarda poi la correlazione scelta monarchica, ideologia politica conservatrice, opzione repubblicana, fede progressista; così si può inferire che a favore della prima ipotesi si siano pronunciati anche elettori di sinistra e, a vantaggio dell'altra, sostenitori della destra (Mura, Colombo).

La variabile territoriale non solo è determinante nella valutazione dei risultati elettorali ma apre una serie di questioni metodologiche di indubbio rilievo. Come viene giustamente posto in evidenza in alcuni saggi, i confini politici ed amministrativi non corrispondono sempre a quelli fisici e geografici. Perciò viene opportunamente sottolineato che la Lombardia non è una regione "naturale" (ma nemmeno "politica", vista la disomogeneità al suo interno dell'andamento elettorale) e non ha neppure una sua precisa identità territoriale. Si comprende allora perché il voto (referendario, e non solo) si "spalmi" in maniera molto differenziata a seconda delle province indagate, con alcune che sembrano più affini a quelle ad egemonia conservatrice ed altre più assimilabili a quelle a prevalente orientamento progressista (Colombo). Lo stesso vale per le Alpi sud-occidentali (a cavallo 
tra Piemonte e Liguria) dove il perimetro delle circoscrizioni amministrative confligge con la diversa articolazione geografica e identitaria, che caratterizza numerose valli e diverse aree di confine statale e sovra-provinciale (Celi). Insomma, non si può non tener conto di questa discrasia nel momento della formulazione di ipotesi interpretative delle statistiche elettorali. Ma il territorio dello Stato non è solcato solo da confini amministrativi ma è ritagliato pure da quelli ecclesiastici, in specie dall'area di "giurisdizione" delle diocesi. Ed anche in questo caso il risultato della votazione segue un andamento che si distacca da quello imputabile unicamente all'appartenenza ad una determinata Provincia o collegio elettorale (Colombo, Mura, Tonietto).

Il secondo aspetto da mettere in risalto concerne il rapporto fra le diverse elezioni che coinvolgono finalmente il Paese dopo il lungo periodo del regime fascista e che si celebrano nell'arco di pochi mesi; ad esse partecipano per la prima volta le donne e molti giovani uomini, che sperimentano così una nuova, e per loro sconosciuta, esperienza di partecipazione democratica. Ciò che si ricava da alcuni contributi del libro è la non "meccanica" sovrapponibilità dei risultati del triplice voto, dovuta a variabili differenti (ad esempio, voto nei capoluoghi o nei comuni più piccoli), o alla maggiore, o minore, importanza attribuita allo scontro referendario rispetto alla scelta dei consiglieri comunali o dei membri dell'Assemblea Costituente. Ecco allora, a seconda dei casi esaminati, profili di continuità o discontinuità nel voto espresso nel 1946, ma anche - in taluni testi - in rapporto a quello risultante dalle ultime elezioni del primo dopoguerra, tenutesi nel 191921. Le elezioni amministrative pongono però non poche difficoltà interpretative e valutative ove si tenga presente, ad esempio, il diverso sistema elettorale adottato nei comuni, in base alla loro entità demografica, o il disagevole reperimento dei dati concernenti i voti ottenuti nelle singole amministrazioni municipali (Forcellese, Schininà, Tentoni, Colombo, Bertolotti).

A prescindere dai risultati, e dalle più o meno sofisticate elaborazioni statistiche in merito, le elezioni comunali mettono in risalto, come emerge da alcuni contributi, due aspetti "politici" di non secondaria importanza. Si è ricordato che la precedenza data alla consultazione locale (primaverile) rispetto a quella politica finiva per pregiudicare ogni eventuale riforma dell'assetto delle autonomie locali, ritenendosi sufficiente, nel nuovo assetto istituzionale democratico, un semplice ripristino del meccanismo elettorale, abolito dal fascismo, mentre si manteneva pressoché inalterato, nel suo complesso, l'ordinamento normativo ereditato dall'età liberale e sostanzialmente conservato nel ventennio, ad esempio, in tema di controlli statali e di finanza locale (Colombo). Altro aspetto di natura politica da non sottovalutare è l'intento che sarebbe stato proprio del Ministro dell'Interno Romita di selezionare, opportunamente e strategicamente, i comuni ove far svolgere le elezioni, onde trainare e influenzare il successivo voto referendario a favore della Repubblica. Sul punto le opinioni dei collaboratori del volume non sono peraltro del tutto convergenti, ma non pare lontana dal vero l'interpretazione "partigiana" che, di quello stratagemma, viene data al di là del suo esito finale e della sua reale efficacia (Morese, Tentoni, Colombo).

Come era del resto prevedibile, sullo sbocco del voto referendario si possono individuare altri elementi di sicura rilevanza e di comprovata incidenza. Non poteva mancare l'influsso della cultura politica, o meglio delle due "sub-culture" di matrice cattolica o di valenza progressista, né quello della struttura socio-economica dei singoli territori, anche se non sempre quelli più "attivi" si dimostrano ovunque in sintonia con le 
tendenze repubblicane. La variabile politica esercita, insomma, un ruolo non marginale e altrettanto si può dire, ad esempio, di una memoria "negativa" della Resistenza o di una sua sostanziale assenza (come in Sardegna), ovvero di una sua accettazione strumentale (vedi il Piemonte), o ancora di un diffuso timore per una possibile affermazione del partito comunista; tutti fattori che favoriscono una scelta "continuista" a vantaggio della Monarchia (Colombo, Forcellese, Mura, Celi, Tonietti).

Quanto al modello economico, non difettano correlazioni significative, e tendenzialmente univoche, con la presenza di forti e tradizionali componenti operaie o con le peculiarità, anche giuridico-contrattuali, del mondo del lavoro contadino (piccola o grande proprietà, mezzadria, colonìa) (Celi, Sellaroli e Zanibelli). C'è pure un ulteriore e innovativo aspetto che viene in luce dai lavori inseriti in questo volume; si tratta dell'influenza, di lungo periodo, di precedenti e persistenti culture politiche, di legami ancestrali di fedeltà dinastica, di ininterrotti sentimenti di deferenza nei confronti di nobili e notabili locali. Si pensi al perdurante ricordo, e conservazione, di tradizioni garibaldine, repubblicane e laiche di ascendenza risorgimentale (Forcellese, Schininà, Mura, Morese). Né può stupire il coinvolgimento del clero, quasi sempre in funzione di una difesa della Casa Reale, che si registra sia nell'attività pastorale che nella propaganda condotta, fra l'altro, attraverso i periodici diocesani. Ed è significativo constatare come nel mondo cattolico e, in specie, all'interno del partito che, in larga misura, lo esprime e lo tutela, la DC, si verifichi persino uno scollamento tra i vertici, apertamente repubblicani, e la base, maggiormente fedele alla gerarchia, sensibile nei confronti dei suoi pronunciamenti e rispettosa delle sue raccomandazioni (Colombo, Mura, Tonietti).

Il riferimento al clero ci può condurre al problema, ben noto in sede storiografica ma non ancora convenientemente esplorato, del voto femminile e della, presunta, sua permeabilità all'influenza dei pastori della Chiesa cattolica. Anche il curatore del volume osserva che, sul punto, molto resta da indagare e in ciò non aiuta certo il carattere "anonimo" e, qualitativamente, "asessuato" dei risultati elettorali. A ben vedere occorrerebbe inoltre indagare, per così dire, su una possibile "frattura" inter-familiare, vale a dire sulla effettiva convergenza del voto delle donne su quello, suggerito o imposto, dai mariti, essendosi verificati sicuramente non pochi casi di voto "disgiunto", ed opposto, per disparità di "genere".

Si è ricordato, all'inizio, che il volume contiene due contributi per così dire "eccentrici" rispetto a quelli da cui ho tratto le osservazioni precedenti. Il primo, dovuto a due economisti, tenta di costruire un modello statistico-matematico che, applicato su una macro-area territoriale - nel caso di specie la Maremma (a cavallo tra Toscana e Lazio) e, grazie anche all'impiego del noto coefficiente (di Gini) e dell'indice di ruralità (Iru), cerca di evidenziare il ruolo del substrato economico-sociale (in particolare, la tipicità fondiaria) nella distribuzione del voto referendario. Dunque la prevalenza della grande proprietà terriera ( la c.d. "land inequality") quale variabile esplicativa - non strettamente politica - anche di un comportamento elettorale che, nel caso considerato, certifica una netta preferenza per la scelta repubblicana. Si tratta ancora di un esperimento, realizzato su una scala rilevante ma comunque circoscritta, e che potrebbe essere replicato in altri contesti territoriali di area vasta in modo altrettanto proficuo.

Incentrato, invece, sui canoni propri della politologia storica è il lavoro condotto sulle già citate sub-culture politiche, la cattolica e la "marxista", per verificare come esse agiscono in due ambiti tipici, e "topici": il Veneto e la Toscana. Dopo una lunga esposizione 
della letteratura scientifica esistente in oggetto, con i dovuti rimandi ad autori ben noti, fra i quali Weber, Bendix, Hintze, Moore, Rokkan, e le puntuali citazioni dei numerosi studi italiani condotti sinora sulle culture politiche territoriali, gli autori, utilizzando altresì i concetti esplicativi di "civicness" e di "capitale sociale", precisano gli indicatori da loro prescelti, e impiegati, per individuare e circoscrivere le due sub-culture in tali contesti geografici. A questo scopo entrano in gioco il cleavage centro/periferia; la presenza di reti associative; il senso di appartenenza territoriale; la continuità del sistema politico locale. Alla fine, tuttavia, lo schema politologico viene applicato su pochi dati elettorali e dunque l'influenza delle sub-culture sulle dinamiche del voto, per quanto sicuramente dimostrata, affiora solo in due ambiti significativi ma ancora limitati, se si pensa al suo prevedibile manifestarsi in altre zone del Paese (l'Emilia-Romagna o il Piemonte) dalla pronunciata dicotomia politico-ideologica.

Voglio infine segnalare un aspetto peculiare, benché secondario rispetto alle riflessioni sinora svolte, nella ricerca di elementi che possono aver condizionato, in una direzione o in un'altra, la scelta dei cittadini nel referendum istituzionale. Secondo il Partito sardo d'azione, l'alternativa Monarchia-Repubblica avrebbe avuto rilevanti conseguenze persino sull'adozione del futuro modello amministrativo (accentrato o decentrato) e l'appoggio all'opzione repubblicana avrebbe facilitato il riconoscimento alla Sardegna di un'autonomia “speciale". I socialisti dell'isola, da parte loro, si battevano contro l'ipotesi monarchica anche per il rigido modello "uniformatore" e statalista che i Savoia avevano imposto in tutta Italia e che aveva causato gravi danni allo sviluppo sociale ed economico dell'isola. Osservazioni, queste, che trovavano sicuramente ascolto in una Regione, come la Sardegna, dove i sentimenti autonomistici, se non separatistici, erano molto forti e radicati (Mura). Similmente in Piemonte e in Liguria, a sostegno del voto repubblicano spingeva pure una forte tendenza autonomistica e federalistica, osteggiata peraltro dal PCI e condivisa invece dal Partito d'azione (Celi).

Un'ultima considerazione, a dimostrazione dell'utilità di questo lavoro non solo a livello accademico ma anche dell'opinione pubblica meno avvertita e della didattica della storia in ambito scolastico, riguarda la puntuale smentita, contenuta nel testo iniziale di Forcellese e formulata pure sulla base di una accurata analisi dei dati elettorali, delle antiche ma ricorrenti e mai sopite polemiche sulla effettiva vittoria dello schieramento repubblicano. Basti pensare che molti anni fa, sia pure con un intento didascalico, e per confutare le stesse teorie cospirative filo-monarchiche, la RAI aveva trasmesso una docufiction, curata, se non erro, dal noto giornalista Giovanni Minoli, in cui si adombrava l'esistenza di una congiura ai danni della Monarchia e solo in chiusura della trasmissione si svelava l'artificiosità e falsità delle tesi ivi sostenute. In un periodo di fake-news e di continue manipolazioni della storia a fini politici, ben venga dunque questa preziosa e documentata confutazione. Ma, come risulta anche dalle riflessioni sinora svolte, è tutto il volume che si raccomanda alla lettura non soltanto degli esperti e degli studiosi, ma di tutti i cittadini consapevoli e curiosi.

Piero Aimo 